\title{
LO QUE DEBEMOS TENER EN CUENTA ANTES DE DISEÑAR PROCESOS DE APRENDIZAJE
}

Luis Bretel B.

Este documento pretende responder, desde la revisión bibliográfica de la literatura pedagógica y de la psicología de la educación contemporánea y de la manera más simple y sintética posible, a las siguientes preguntas: ¿Qué es aprender? ¿Qué tipos de aprendizaje podemos tener? ¿Cómo aprendemos cada tipo? Y ¿Qué tiempo requerimos para hacerlo? Esperamos que su contenido pueda ayudar a tomar decisiones acertadas sobre cómo diseñar cursos que hagan posible aprendizajes exitosos.

aprendizaje capacitaci $\tilde{\mathrm{A}}^{3} \mathrm{n}$ proceso educativo 


\section{¿Qué es aprender?}

Es re-estructurar, por asimilación y acomodación, nuestras estructuras de pensamiento....

Desde Piaget, pasando por Ausubel y llegando hasta los pioneros de la neuroeducación, existe consenso respecto a la afirmación de que el aprendizaje es un proceso por el que se van enriqueciendo nuestras estructuras mentales, conceptuales y neuronales, lo que nos permite comprender y vivir en el mundo; del mismo modo puede afirmarse que aprender es ampliar y enriquecer los procedimientos con los que podemos actuar en el mundo o las actitudes y valores que nos permiten ir siendo y haciendo de maneras determinadas. Desde otra perspectiva, puede decirse que el aprendizaje es el proceso por el que se desarrollan nuestras capacidades, que pueden llegar a convertirse en habilidades, destrezas o hasta en competencias.

Pero, se vea desde una u otra perspectiva, el aprendizaje no es un proceso de simple adición, sino más bien uno mucho más complejo de re-estructuración o, desde la perspectiva de Piaget, de asimilación y de acomodación. Ello implica que la variación que produce el conocimiento, en el sujeto que aprende, no es cuantitativa sino que por el contrario "... tiene lugar un cambio de la propia estructura de conocimiento a través de la reordenación de esquemas, de manera que adquiere así más importancia la comprensión que la acumulación; es decir, se produce una variación principalmente cualitativa." (Sánchez Iniesta, 1995. Pag. 20)"

\section{... a partir del conflicto cognitivo...}

Debemos tener en cuenta que nadie se encuentra frente al nuevo conocimiento como "tabula rasa" (mesa vacía), carente de toda información y de toda idea preconcebida acerca de lo que aprenderá. Todas las personas, desde muy pequeñas, tenemos nuestras propias asunciones sobre cómo funciona el mundo o una parte de él. En 
sus años pre-escolares, los niños empiezan a desarrollar comprensiones sofisticadas (ya sean precisas o no) de los fenómenos a su alrededor (Wellman, 1990). Esas comprensiones iniciales pueden tener un fuerte efecto en la integración de nuevos conceptos e información. Algunas veces esas comprensiones son precisas y ofrecen cimientos para construir nuevos conocimientos. Pero, otras veces, son imprecisas (Carey y Gelman, 1991). Los estudiantes, con frecuencia, tienen concepciones erróneas de las propiedades físicas que no pueden ser observadas fácilmente. En letras, las concepciones previas incluyen a menudo estereotipos o simplificaciones, como cuando la historia se comprende como una lucha entre los buenos y los malos (Gardner, 2001).

$\mathrm{Y}$, si esas comprensiones iniciales no llegan a verse comprometidas en el proceso de aprender, es posible que la persona no llegue a comprender los nuevos conceptos e información que se le enseña, o podría aprenderlos para fines de una evaluación pero, fuera del espacio formal de clase, en el "mundo real" seguirá manteniendo sus concepciones previas.

"Con mucha frecuencia los esfuerzos deliberados por adquirir o enseñar conocimientos explícitos tropiezan con los obstáculos que plantean los conocimientos implícitos aprendidos con anterioridad de modo incidental y sin que el aprendiz sea ni siquiera consciente de ello. Ello obliga a reconstruir esos conocimientos implícitos, reflexionando sobre ellos, porque sólo así podremos cambiarlos, sea en nuestras relaciones con los demás o con nosotros mismos, en la adquisición de conceptos o en el desarrollo de nuevas habilidades y estrategias." (Pozo, 1999. Pag. 76)

Por tales razones es importante, para los aprendices de todas las edades, identificar o trabajar con sus comprensiones pre-existentes, pensar en ellas y tener la oportunidad de ver cuáles son las fallas de estas comprensiones. Sólo así el conocimiento científico reemplazará sus comprensiones ingenuas o erróneas. Esto le permitió afirmar a Piaget que, para que el aprendizaje sea posible, es necesario provocar un "conflicto cognitivo", que se origina en la puesta en cuestión de los esquemas y comprensiones previas. Sostiene el autor que para restablecer el equilibrio (cognitivo) producido por el conflicto, es preciso que se den de modo complementario los procesos de asimilación y de 
acomodación. Por el primero (asimilación) incorporamos a nuestra estructura de conocimiento la información que procede del medio, que a su vez se modifica al relacionarse con los esquemas que ya posee cada persona. Luego se contrastan estas teorías personales mediante su aplicación a la realidad y se genera así un proceso de acomodación, por el cual se modifican estas teorías en función de la respuesta obtenida en su aplicación al medio. Para Piaget, el alumno aprende principalmente por descubrimiento en un proceso constante de relación con el medio, a través de un continuo entre la asimilación y la acomodación.

\section{...para conseguir una nueva comprensión...}

Para desarrollar expertise y competencia en un área específica, los aprendices no sólo deben tener una base profunda de conocimiento fáctico sino que, sobre todo, deben tener la oportunidad de aprender comprendiendo. La comprensión implica el establecimiento de conexiones: entre la nueva información y la que ya se conocía y entre los nuevos elementos de información (Brandsford, 2000). La comprensión profunda de una materia trasforma la información fáctica recibida en conocimiento propio y utilizable que sólo surge de la reflexión del sujeto sobre la información, la aplicación de su capacidad de discriminación y el discernimiento respecto de la información, la capacidad de jerarquizar, de ordenar y de maximizar la información (Savater, 2012).

Por esta razón, es tan obvia la diferencia entre los expertos y los novatos en la comprensión de nueva información: Los expertos descubren patrones, relaciones o discrepancias que no son aparentes para los novatos. Los expertos no necesariamente tienen una mejor memoria global que las otras personas, pero su comprensión conceptual les permite extraer el nivel de significado de la información que no es evidente para los novatos, y esto les ayuda a seleccionar, identificar y recordar información relevante (Pozo, 2008).

Sin embargo, es evidente que a lo largo de nuestra vida debemos aprender muchas cosas que no necesitamos comprender o cuya comprensión es innecesaria o absurda (un número telefónico, una dirección, etc.) o muy compleja para la necesidad inmediata de 
ese conocimiento (Si estoy aprendiendo potenciación y sólo me interesa saberla para elevar algunos números a una potencia determinada, no necesito comprender por qué un número elevado a la potencia 0 es igual a 1; si estoy aprendiendo a usar una calculadora para utilizarla de apoyo en un trabajo numérico, no necesito comprender por qué la secuencia de teclas a apretar para guardar una cifra en algunas calculadoras es distinta a otra). Sin embargo, es necesario tener en cuenta que tales aprendizajes no serán nunca significativos y duraderos. Razón por la cual debemos asegurarnos que el aprendiz tenga y pueda recurrir a ayudas némicas (una agenda, una tabla de fórmulas, una guía de operación) cuando requiera recordar esa información que se aprendió sin comprender y sin producir aprendizajes significativos. Y. en sentido inverso, poder determinar sobre qué nueva información es indispensable que se produzcan, en alumno, aprendizajes significativos y asegurarse que efectivamente se produzcan, es decir, que el alumno pueda explicar lo aprendido con sus propias palabras, que pueda ejemplificarlo, que pueda proponer relaciones entre la nueva información y la que ya poseía, etc..

\section{... con un esfuerzo metacognitivo que haga posible la transferencia.}

Un hallazgo clave en las investigaciones sobre el aprendizaje es el descubrimiento de que organizar la información en un marco conceptual propio permite una mayor "trasferencia", es decir, permite que el estudiante aplique lo que ha aprendido a las nuevas situaciones y que aprenda información relacionada al tema de manera más rápida (Claxton).

En la investigación con expertos se ha hecho evidente también, que ellos monitorean cuidadosamente su propia comprensión, tomando nota sobre cuando se requiere información adicional, sobre si esta nueva información es consistente con lo que ya conocían y qué analogías podrían extraerse que podrían incrementar su comprensión. Estas actividades de monitoreo metacognitivo son un componente importante de lo que se llama un expertise adaptativo (Bransford). 
Por ello se afirma que un enfoque "metacognitivo" de enseñanza puede ayudar a los estudiantes a aprender y a tomar el control de su propio aprendizaje, tanto al definir las metas de aprendizaje como al monitorear su progreso para alcanzarlas. Por esto es indispensable enseñar estrategias metacognitivas dentro de las materias que los estudiantes están aprendiendo, teniendo en cuenta que estas estrategias no son genéricas y que los intentos por enseñarlas como genéricas o al margen de las materias específicas pueden llevar al fracaso en la trasferencia (White y Frederickson, 2000). Hay suficiente evidencia empírica acerca de que aprender estrategias metacognitivas en el contexto en el que se aprende física (White y Frederickson, 2000), composición escrita (Scardamalia y col., 1984) y matemáticas (Schoenfeld,1991), mejora considerablemente el nivel de comprensión que alcanzan los estudiantes. Y se ha hecho evidente también que las prácticas metacognitivas incrementan el grado en el que los estudiantes trasfieren lo que saben a nuevos contextos y situaciones. (Scardamalia y col., 1984; Schoenfeld, 1991).

De las diversas y diferentes definiciones de aprendizaje Pozo (1999) extrae tres rasgos prototípicos del buen aprender: implica un cambio duradero, porque ha implicado la reestructuración de las estructuras de pensamiento y comprensión; es transferible a nuevas situaciones $\mathrm{y}$ es consecuencia directa de la práctica realizada por el propio aprendiz y no sólo de la "exposición" al o del nuevo conocimiento. Lo que se aprende debe poder utilizarse en otras situaciones. Y ésta, la transferencia, no es un proceso automático que se produzca de modo inevitable siempre que aprendemos algo. Wertheimer (1991) diferencia el aprendizaje reproductivo, basado en la aplicación rutinaria de aprendizajes anteriores, del aprendizaje productivo, que implica comprender lo aprendido, a través de la construcción de una estructura. El aprendizaje es siempre producto de la práctica. Pero es el tipo de práctica y no la cantidad de práctica lo que identifica al buen aprendizaje. La práctica repetitiva produce aprendizajes más pobres o limitados que la práctica reflexiva. “Por ejemplo, en la formación de expertos, Glaser (1992, pág. 74) considera que una práctica reflexiva, basada en principios teóricos y no en una mera repetición, 'permite comprender lo que se está haciendo, recuperarse con rapidez y elegancia de los errores y aprovechar las oportunidades para obtener soluciones 
y descubrimientos más armoniosos y precisos. Ser experto se convierte así en algo más que pura eficiencia y los conocimientos, a medida que se adquieren, se convierten en un motivo para aprender de la experiencia e interrogarla, de forma que acaban por reorganizarse abriendo el camino a nuevas ideas y acciones'."(Pozo. 1999. Pag. 83)

\section{¿Qué tipos de aprendizaje podemos tener?}

\section{Aprendizaje Conceptual}

Aprendizaje Conceptual consiste en la incorporación de datos, conceptos y principios a la estructura mental del sujeto. Este aprendizaje permite describir, entender, explicar, fundamentar y proyectar la acción. Como afirmábamos antes, hay muchos hechos y datos que debemos incorporar a nuestra memoria sin que deban tener un significado y requieran comprensión, ya que debemos repetirlos literalmente, por ejemplo, nombres de personas, de calles, de ciudades, etc. Pero, cuando aprendemos conceptos nuevos, que nos permiten atribuir significado a los hechos interpretándolos dentro de un marco conceptual, no se trata sólo de adquirirlos como hechos o datos yuxtapuestos, uno al lado del otro, sino comprender por qué se relacionan entre sí y no de otra forma (Pozo, 2008).

La comprensión implica conectar o asimilar una información nueva a conocimientos previos. Pero ya se ha dicho que el aprendizaje conceptual implica cambio y reestructuración de los conocimientos previos, con el fin de construir nuevas estructuras conceptuales que permitan integrar tanto los conocimientos anteriores como la nueva información presentada y que este cambio sólo se producirá si ha ocurrido un conflicto cognitivo que confronte teorías o explicaciones distintas con respecto a un mismo suceso. (Pozo, 2008). Lograr, finalmente, que el aprendizaje sea significativo implica que lo aprendido se relacione sustancialmente con los conocimientos previos (la estructura cognitiva) del aprendiz. 


\section{Aprendizaje procedimental}

Aprendizaje procedimental es el que consiste en aprender a "hacer algo", no sólo comprenderlo o decirlo. Este aprendizaje implica la adquisición de técnicas o estrategias de acción y el desarrollo de capacidades hasta constituirse en "secuencias de habilidades o destrezas más complejas y encadenadas que un simple hábito de conducta." (Pozo, 1999. Pag. 98).

Entendemos aquí por habilidad el nivel específico de desarrollo de alguna capacidad, alcanzado mediante un proceso de aprendizaje y entrenamiento. Capacidad, por otra parte, es cada una de las posibilidades iniciales y potenciales de desarrollo que poseen los seres humanos (salvo excepciones patológicas) y que pueden ser desarrolladas mediante el aprendizaje, a partir de experiencias cristalizantes, o inhibidas por experiencias paralizantes (Gardner, 1987). Algunas de estas capacidades pueden ser por ejemplo: la capacidad de emplear palabras en la comunicación oral o escrita, la capacidad de emplear números y contar, la capacidad para distinguir patrones lógicos, la capacidad para percibir el mundo visual y espacial, la capacidad para controlar los movimientos del propio cuerpo, la capacidad para manipular objetos, etc.. Todas estas capacidades las poseemos al nacer por el solo hecho de haber nacido con un cerebro humano, pero deben ser desarrolladas mediante procesos de aprendizaje para constituirse en habilidades o destrezas.

Haber adquirido una habilidad implica la posibilidad de hacer bien algo, producto de la práctica y la experiencia propias, así como la posibilidad de transferir ese saber a situaciones distintas a aquellas en las que se produjo su aprendizaje. Por ejemplo: La habilidad de formalizar matemáticamente, mediante números, variables y funciones, hechos o fenómenos cotidianos, se puede adquirir gracias a que se poseen las capacidades de emplear números y de razonar lógicamente y sólo se sabe que se adquirió si se puede hacer uso de ella en diversas situaciones problemáticas de la vida real. 


\section{Técnico}

El aprendizaje procedimental puede ser meramente técnico, por ejemplo cuando se aprenden secuencias de acción que deben ser realizadas de modo rutinario con el fin de alcanzar siempre el mismo objetivo. Éstas pueden ser secuencias de acción simples, aprendibles por exposición a un modelo o a un programa de refuerzo, o complejas ya que requieren encadenamientos de acciones complejas que exijen un cierto entrenamiento explícito, basado, eso sí, en un aprendizaje por repetición, o asociativo, que debe concluir en una automatización de la cadena de acciones, con el fin de que la ejecución sea más rápida y certera, al tiempo que menos costosa en recursos cognitivos.

El aprendizaje técnico es muy eficaz cuando nos enfrentamos a ejercicios y tareas rutinarias, siempre iguales a sí mismas, pero no así cuando la situación varía en algún elemento importante, ya que en dichos casos no basta con dominar la técnica, hay que saber también modificarla sobre la marcha, para poder adecuarla a las nuevas condiciones. (Pozo, 1999) Si siempre serán usadas en las mismas condiciones bastará el entrenamiento, pero si se espera que se usen en una diversidad de condiciones, el entrenamiento debe estar acompañado por una compresión de cuándo, cómo y por qué usarlas, lo que hace que deje de ser éste un aprendizaje técnico para convertirse en un aprendizaje estratégico.

\section{Estratégico}

El aprendizaje estratégico o de estrategias para planificar, tomar decisiones y controlar la aplicación de las técnicas, así como para adaptarlas a las necesidades específicas de cada tarea, no se logra por procesos asociativos o repetitivos, sino por procesos de reestructuración de la propia práctica, producto de una reflexión y toma de conciencia sobre lo que hacemos y cómo lo hacemos (Wertheimer, 1991).

Aprendemos estrategias a medida que intentamos comprender o conocer nuestras propias técnicas y sus limitaciones, y ello requiere también que hayamos aprendido a tomar conciencia y reflexionar metacognitivamente sobre nuestra propia actividad y cómo 
hacerla más efectiva. (Pozo, 2008). No es posible pues aprender estrategias sólo por entrenamiento, porque su uso supone la aplicación organizada y controlada de técnicas y recursos disponibles.

\section{Aprendizaje actitudinal}

Las actitudes se suelen definir como disposiciones afectivas y racionales que se manifiestan en los comportamientos, o como tendencias a juzgar las cosas, las personas, los sucesos o situaciones y a actuar en consonancia con dichas evaluaciones. En las actitudes es usual distinguir un componente conductual (forma determinada de comportarse), rasgos afectivos y una dimensión cognitiva (no necesariamente consciente). Las actitudes no son innatas, por el contrario, son aprendibles y modificables. Se adquieren en la experiencia y en la socialización y son relativamente duraderas y resistentes.

En tal sentido, el aprendizaje actitudinal, que consiste en la modificación o adquisición de actitudes, se logra con mayor eficacia por exposición a modelos o provocando situaciones de conflicto que hagan evidentes las contradicciones entre el juicio, el sentimiento y la acción. Los aprendices tienden a adoptar en su aprendizaje actitudes congruentes con los modelos que han recibido, pero no cualquiera de ellos, sino con mayor probabilidad aquellos con los que se identifiquen, con los que creen o compartan como parte de una identidad común. (Pozo, 1999).

El cambio de actitudes sólo es posible si el aprendiz es sometido a situaciones de conflicto cuya resolución requiera modificar las actitudes hasta ahora mantenidas. La introducción de conflictos o inconsistencias desestabiliza las actitudes y fomenta el cambio, ya que en general, esos desequilibrios son desagradables cuando se perciben y cuando se es consciente que se está en medio de ellos.

Según Pozo, son dos los tipos de conflicto que pueden desencadenar aprendizajes actitudinales: el "conflicto sociocognitivo" o la "disonancia cognitiva". El primero es el 
conflicto que se produce entre las propias actitudes y los valores del grupo de referencia. Se produce cuando nos damos cuenta que el grupo con el que nos identificamos tiene actitudes diferentes a las nuestras. Si esto se produce el cambio o aprendizaje actitudinal es relativamente más facil de conseguir. Por otra parte la "disonancia cognitiva" es el conflicto interno que se produce cuando descubrimos la existencia de una contradicción entre nuestras actitudes y valores y nuestras propias conductas. Cuando caigo en la cuenta que mi conducta no corresponde o no es coherente con mis creencias y valores manifiestos.

\section{¿Qué tiempo es necesario para aprender?}

Un primer tiempo a tener en cuenta, cuando se quiere lograr aprendizajes, es el tiempo que necesitamos los docentes para identificar lo que los aprendices saben o no respecto a lo que queremos que aprendan, ya que sin saber esto difícilmente encontraremos cómo provocar el conflicto conginitivo, necesario para producir aprendizajes.

\section{Tiempo para motivar ...}

Es indispensable prever el tiempo necesario para generar y mantener la motivación por el nuevo aprendizaje. Dada la complejidad de los procesos mentales y cognitivos involucrados en el proceso de lograr aprendizajes significativos, es evidente que la primera tarea del docente, si quiere lograrlos, es asegurar que se haya producido la suficiente movilización afectiva y volitiva del aprendiz, como para que esté dispuesto a aprender significativamente, es decir a realizar el esfuerzo mental que ello supone, y para que pueda sostenerse en el esfuerzo. Es claro que no todos los alumnos se sienten igualmente motivados para aprender o para aprender determinadas cosas, pero también lo es que si no logramos motivarles el aprendizaje no será posible y todos perderemos nuestro tiempo.

$$
11 \text { Page Vol 1, No2/2013 ABCResearch Ale Rt }
$$


No pretendemos aquí profundizar sobre los tipos de motivación (intrínseca y extrínseca) y sobre todo aquello que puede operar como motivadores, sólo afirmar que los móviles extrínsecos (el ascenso, la nota, una recompensa, un castigo, etc.) no son suficientemente potentes como para lograr procesos sostenidos de aprendizaje significativo, mientras que los móviles intrínsecos (interés por el tema. curiosidad, deseo de saber más respecto a algo, etc.) lo son mucho más, aunque mejores resultados se alcanzan si se logra una combinación de ambos.

También, es importante señalar que la motivación no corresponde, como muchas veces se ha malentendido, con una etapa inicial y cancelatoria en el proceso de aprendizaje, ya que no basta que el alumno esté motivado antes de iniciar la actividad de aprendizaje, aunque luego la actividad misma sea desmotivadora y desmovilizadora. Por el contrario, es indispensable caer en la cuenta que en el momento en que la motivación por aprender no sea alimentada el proceso puede interrumpirse.

Por otra parte, debemos caer en la cuenta que la motivación afecta la cantidad de tiempo que las personas estamos dispuestas a dedicar para prender. Los humanos estamos motivados a desarrollar competencias y a resolver problemas $y$, aunque las recompensas y castigos extrínsecos claramente afectan la conducta, las personas también trabajamos arduamente por razones intrínsecas. Sin embargo, los retos deben estar a un nivel adecuado de dificultad para ser y permanecer motivantes: las tareas que son demasiado fáciles se hacen aburridas, mientras que las tareas que son demasiado difíciles causan frustración.

Por otra parte, debe tenerse en cuenta que la tendencia de los aprendices para persistir encarando dificultades es bastante afectada por si ellos se orientan "al desempeño" o "al aprendizaje" (Dweck, 1989). Los estudiantes que se orientan al aprendizaje prefieren los retos nuevos y quienes se orientan al desempeño están más preocupados por no cometer errores que por aprender. 
Es importante recalcar que la comprensión es también importante en la motivación, además de para lograr aprendizajes significativos, ya que la no comprensión no sólo produce aprendizajes mecánicos, sino que desmotiva y desmoviliza al aprendiz, porque le hace sentirse ineficaz y torpe para aprender, además de no dejarle más alternativa que aprender por obligación y por repetición mecánica algo que no comprende.

Finalmente, es necesario señalar que los aprendices de todas las edades están más motivados cuando pueden ver la utilidad de lo que están aprendiendo y cuando pueden utilizar esa información para hacer algo que podría tener o tiene un impacto en los otros, especialmente en los más cercanos.

\section{Tiempo para provocar conflicto cognitivo y conseguir su solución: las nuevas comprensiones.....}

Explicábamos anteriormente que si los aprendices no llegan a encontrarse en una situación de desequilibrio y sus esquemas de pensamiento no entran en contradicción, difícilmente se lanzarán a buscar respuestas, se plantearán interrogantes, investigarán, descubrirán, es decir: aprenderán. De esta manera el conflicto cognitivo no sólo se convierte en ese motor afectivo y motivacional de nuevos aprendizajes, sino en la garantía de que efectivamente las estructuras de pensamiento se verán modificadas, porque ya no pueden seguir siendo las mismas.

Este concepto le da mayor sentido a la necesidad de conocer los saberes previos (en tanto estructuras cognitivas previas) de los alumnos y plantea un mayor reto creativo al docente: ¿cómo lograr que entren en colisión los esquemas cognitivos de mis alumnos? Sin embargo, en la respuesta a esta pregunta se encuentra con seguridad no sólo la clave afectiva y emocional que garantizará la motivación durante todo el proceso de aprendizaje, sino la seguridad de que el alumno no aprenderá sin haber hecho un gran esfuerzo por comprender lo que está aprendiendo y que, por tanto, la nueva información será efectivamente asimilada (en el ligeramente diferente sentido en que Piaget y Ausubel 
conciben este proceso) en sus estructuras de pensamiento, reestructurándolas.

Es tan importante tener presente lo anterior que podríamos afirmar que sin haber producido conflicto cognitivo, difícilmente conseguiremos nuevos aprendizajes en nuestros alumnos.

\section{Tiempo para la práctica y la transferencia}

Necesitamos tener claro cuál es el nivel de aprendizaje o expertise que esperamos que los aprendices alcancen respecto a la materia de su aprendizaje, porque debemos ser muy realistas sobre la cantidad de tiempo que toma aprender una materia compleja y llegar al nivel de aprendizaje esperado. Por ejemplo, se ha estimado que los campeones mundiales de ajedrez requieren entre 50,000 y 100,000 horas de práctica para llegar a ese nivel de expertise (Ericsson, 1996). En todas las áreas del aprendizaje, el desarrollo de expertise sólo se logra invirtiendo grandes cantidades de tiempo en prácticas y revisiones, además de la cantidad tiempo que tome aproximarse y comprender inicialmente el material.

Intentos por cubrir demasiados temas de manera muy rápida podrían dificultar el aprendizaje y la transferencia subsiguiente, porque los estudiantes o aprenderían solo conjuntos aislados de hechos que no han logrado organizar o conectar (en su propia mente) o no podrían comprender toda la información porque no tienen suficiente conocimiento especifico y tiempo como para hacerlo significativo. Dar a los estudiantes el tiempo para aprender también implica dar el tiempo suficiente para que procesen la información. El aprendizaje no puede ser apurado, la actividad cognitiva compleja requiere tiempo.

Es claro que distintas maneras de utilizar uno mismo su tiempo tiene efectos distintos en el aprendizaje y la transferencia. Por ejemplo, el aprendizaje es más efectivo cuando las personas se involucran en "practicas deliberadas" que incluye un monitoreo activo de las propias experiencias de aprendizaje (Ericsson y otros, 1993). El monitoreo 
involucra los intentos para buscar y utilizar información sobre el progreso de cada uno.

La retroalimentación es otro elemento importante para un aprendizaje exitoso. Pero es absolutamente distinta la retroalimentación que señala progreso al memorizar hechos y formulas de aquella que señala el estado de la comprensión del estudiante. Además, los aprendices necesitan una retroalimentación sobre el grado en el cual conocen y sobre cuándo, dónde y cómo utilizar el conocimiento que están aprendiendo.

Por otra parte, debemos tener en cuenta que la transferencia también se ve afectada por el contexto del aprendizaje original: las personas pueden aprender en un contexto y aun así no poder transferir a otros contextos. La investigación indica que la transferencia hacia otros contextos es bastante difícil cuando se le enseña a un sujeto solamente en un solo contexto en vez de en múltiples contextos diferentes (Bjork \& Richardson-Klavhen, 1989). Por el contrario, cuando se enseña en varios contextos y se incluyen ejemplos que demuestran una amplia aplicación de lo que está siendo enseñado, las personas tienden a abstraer las características relevantes de los conceptos y a desarrollar una representación flexible del conocimiento (Barnett \& Ceci, 2002).

Una manera de enfrentar la falta de flexibilidad es pedir a los aprendices a resolver un caso especifico y luego darles un caso similar, adicional. La meta es ayudarles a abstraer principios generales que los lleven a transferencias más flexibles (Barnett \& Ceci, 2002). Una segunda manera de mejorar la flexibilidad es permitiendo que los estudiantes aprendan en un contexto específico y luego ayudarles a involucrarse en problemas del tipo “que pasa si.." Diseñados para incrementar la flexibilidad de su comprensión. Se les podría preguntar: ¿Que pasaría si se cambiase esta parte del problema o esta otra?. Una tercera manera es generalizar el caso de manera que se les pida a los aprendices que creen una solución que se aplique no sólo a un problema en particular sino a todos los problemas relacionados.

También es posible mejorar la transferencia ayudando a los estudiantes a estar más conscientes de sí mismos, como aprendices que monitorean activamente sus 
estrategias de aprendizaje y sus recursos y evaluando sus desempeños específicos. Por esta razón, los enfoques metacognitivos de enseñanza aumentan el grado en el que los estudiantes transfieren lo que aprenden a nuevas situaciones sin necesidad de un inductor explícito.

\section{Referencias bibliográficas}

Ausubel, David (1976). Psicología educativa, un punto de vista cognoscitivo. México D.F.: Trillas.

Barnett, Susan M. y Ceci, Stephen J. (2002) When and Where Do We Apply What We Learn?: A Taxonomy for Far Transfer. Psychological Bulletin de la American Psychological Association, Inc. Vol. 128, No. 4, 612-637. Visto el 2/11/2012 en: http://rapunselshair.pbworks.com/f/barnett 2002.pdf

Bjork, R.A., and A. Richardson-Klavhen. (1989) On the Puzzling Relationship Between Environment Context and Human Memory. Pp. 313-344 in Current Issues in Cognitive Processes: The Tulane Flowerree Symposium on Cognition, edited by $\mathrm{C}$. Izawa. Hillsdale, N.J.: Lawrence Erlbaum Associates.

Bransford, John (Editor). (2001) How people learn. National Research Council, National Academy Press.

Carey, Susan y Gelman, Rochel (1991). The Epigenesis of Mind: Essays on Biology and Cognition. LEA. USA.

Claxton, Guy (2001) Aprender: El reto del aprendizaje continuo. Paidos. España.

Covington, Martin (2000). La voluntad de aprender: Guía para la motivación en el aula (Celina González, trad.). Madrid: Alianza Editorial. 
Dweck, Carol S. (1989) Motivation. En: Foundations for a psichology of education. Pp 87136. Hillsdale: NJ.

Docampo, Domingo. 2001. Educación centrada en el aprendizaje. En: http://etc.uab.cat/usuaris/servei apartat/arxius/3/docampo.pdf. Visto el 2/11/2012. pag.3.

Ericsson, K. A., Krampe, R. T. y Tesch-Römer, C. (1993). The role of deliberate practice in the acquisition of expert performance. Psychological Review, 100, 363-406.

Ericsson, K. A., y Lehmann, A. C. (1996). Expert and exceptional performance: Evidence on maximal adaptations on task constraints. Annual Review of Psychology, 47, 273-305.

Gardner, H. (1987) Estructuras de la mente: La teoría de las inteligencias múltiples. Fondo de Cultura Económica. México.

Gardner, H. (2001) Unschooled Mind: How Children Think and How Schools Should Teach. Basic Books, New York.

Piaget, Jean. (1999) Psicología de la inteligencia. Madrid: Psique.

Pozo Municio, Juan Ignacio (1999). Aprendices y maestros: La nueva cultura del aprendizaje. Alianza Editorial. España.

Pozo Municio, Juan Ignacio (2008). Aprendices y maestros: La psicología cognitiva del aprendizaje. Alianza Editorial. España.

Sánchez Iniesta, T. (1995) La construcción del aprendizaje en el aula. Bs. As. Magisterio de Río de la Plata.

Savater, Fernando. Razón, Filosofía y Educación. En: http://ares.unimet.edu.ve/humanidades/bphu05/lecturas/F.\%20SAVATER.\%20RAZ 


\section{ON,\%20FILOSOFIA\%20Y\%20EDUCACION.pdf 2/11/2012.}

Scardamalia, Bereiter \& Steinbach (1984). Teachability of reflective processes in written composition. Cognitive Science, 8, 173-190

Schoenfeld, A. H. (1991). On mathematics as sense-making: An informal attack on the unfortunate divorce of formal and informal mathematics. In J. F. Voss, D. N. Perkins, \& J. W. Segal (Eds.), Informal reasoning and education (pp. 311-343). Hillsdale, NJ: Erlbaum.

Wellman,Henry M. (1990) The Child's Theory of Mind. MIT Press; Cambridge, MA, USA.

Wertheimer, Max. (1991). Pensamiento productivo. Paidos. España.

White, B., \& Frederiksen, J. (2000) Metacognitive facilitation: An approach to making scientific inquiry accessible to all. En J. Minstrell and E. van Zee (Eds.), Inquiring into Inquiry Learning and Teaching in Science. (pp. 331-370). Washington, DC: American Association for the Advancement of Science. 